\title{
Design and Implementation of FSK Modulation and Demodulation Module Using CPLD
}

\author{
Lihua Wu, Yu Huang \\ College of Science, Harbin Engineering University Harbin, Heilongjiang, China \\ wulihua@hrbeu.edu.cn, huangyu_cge@china.com.cn
}

\begin{abstract}
According to the working principle of digital FSK modulation and demodulation, two kinds of circuit schemes based on CPLD and single chip are compared. The circuit module of FSK modulation and demodulation based on CPLD is designed, and CPLD is programmed by Quartus II software to carry out the function of FSK modulation and demodulation. The single chip ATMEGA16 is programmed by IAR FOR AVR software to carry out the function of bit synchronization, and it can communicate with outside by SPI interface. Both simulations and experimental tests prove the exactness of design through building up the actual circuit.

Index Terms - FSK modulation and demodulation, CPLD, single chip, bit synchronization
\end{abstract}

\section{Introduction}

Digital modulation and demodulation is an important topic of modern communication. In the communication system, base-band digital signal includes abundant low-frequency signal. It is essential that the power spectrum is moved to higher carrier frequency via carrier modulation of digital signal in order to transfer digital signal far in finite band-width channel or fiber. FSK(frequency shift keying) is a prior information transfer manner for its advantages in noise resistance, transfer distance and bit error rate, and therefore it is widely applied in medium or low speed data transfer in fading channel[1-2]. In recent years, scholars and corporations did their endeavors in research and popularization of modulator and demodulator. There have been a mass of productions of Bluetooth communication equipment, medical embedded microelectronics device and portable consumer electronics[3-8]. FSK modulation and demodulation can be designed by single-chip or CPLD[1,3]. In the scheme of single-chip, the occupancy rate of resource is higher, for example, an interrupt will happen every other $1 / 300 \mathrm{~ms}$ for ATMEGA16. Because the single-chip deals with interrupt at most time, it is easy to snatch interrupt to make the modulation and demodulation system instable. Generally, the port operation speed of single-chip is at most $1 \mathrm{MHz}$, and its baud rate of FSK signal is unable to very high. However, the port operation speed of common CPLD reaches $120 \mathrm{MHz}$, so the FSK modulation and demodulation using CPLD can be design upper baud rate with the advantage of parallel run of CPLD program and escape of contest in hardware resource. We select the design scheme of FSK modulation and demodulation based on CPLD.

\section{II . System Design}

The schematic chart of working principle for FSK signal produced by one piece of CPLD is shown in Fig.1. The system clock provided by $100 \mathrm{MHz}$ oscillator is used to be local oscillation, which become carrier waves with frequencies f1 and $\mathrm{f} 2$ via the first and second dividers, respectively. The two carrier waves are connected with a gating switch, who selects corresponding carrier wave according to logic input. The carrier wave with $\mathrm{fl}$ will be selected when input is ' 1 ', and carrier wave with $\mathrm{f} 2$ will be selected when input is ' 0 '. FSK signal will be produced through selection of carrier waves with different frequency.

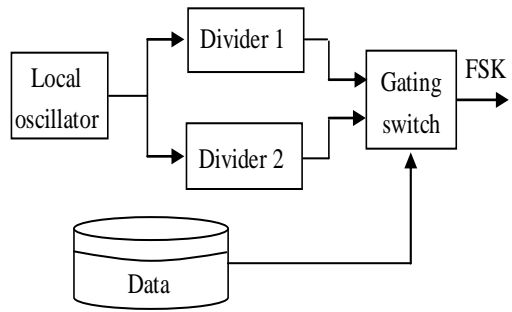

Fig. 1 FSK modulation principle based on CPLD

A zeros-crossing detection method is used for FSK demodulation based on CPLD, which is suitable for digital implementation due to some advantage with simple structure, easy to realize, insensitive to gain fluctuation. The frequency of FSK is determined by the number of it crossing zero voltage axes in unit time. Before transferring to CPLD, FSK is taken out its amplitude through signal processing of limiter and amplification from pre-circuit. The schematic chart for FSK demodulation based on CPLD is shown in Fig.2, where the divider module of CPLD is used to broaden impulse, counter and rectifier modules is used for edge detection. The judging base band signal can be achieved by LPF (low-pass filter) module and the demodulation of FSK comes true.

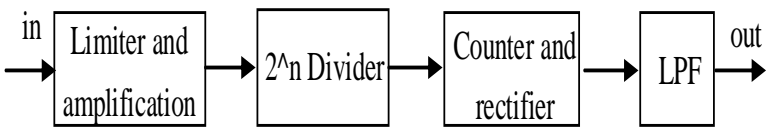

Fig. 2 FSK demodulation principle based on CPLD

The whole circuit chart of FSK modulation and demodulation based on CPLD is shown in Fig. 3 with four modules including power, pre-signal processing, CPLD and single-chip control. A piece of MAX II series CPLD (EPM $240 \mathrm{~T} 100 \mathrm{I} 5 \mathrm{~N}$ ) is used for modulation and demodulation,

and single-chip (ATMEGA16) is for a controller. The serial port is a digital communication system which includes asynchronous bit synchronization, so the demodulation signal can be directly reverted to digital signal by the serial port of 
single-chip and then a SPI interface is set aside to communicate with outside via leaving out bit synchronization of CPLD .

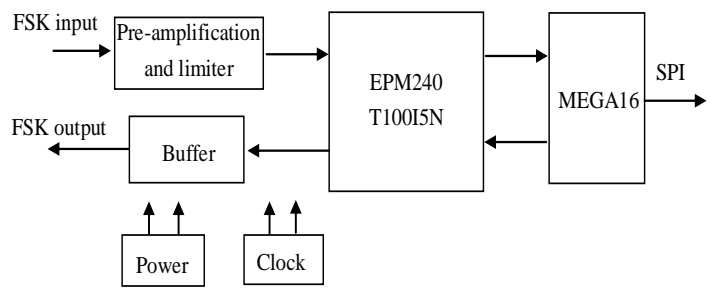

Fig.3 Circuit chart of FSK modulation and demodulation

In the procedure of modulation, single-chip transmits serial data including start bit, stop bit and a parity check bit to CPLD by serial port TXD. FSK can be produced via modulating the base-band signal from CPLD, and more is transmitted through a buffer. The procedure of demodulation is opposite to modulation. Firstly, FSK is introduced to CPLD after limiting and amplifying through pre-processing module. Secondly, FSK amplified is demodulated by CPLD and transmit to single-chip through TXD. Finally, the serial port carries out bit synchronization to pick up data, which is exported via SPI.

\section{III . Design of System Software}

The logical structure of FSK modulation module is designed by VHDL as shown in Fig.4, and the signal of interface is defined as followed. Clk: carrier wave of FSK modulation. It is shaped by 8 and 4 times frequency divider composed of an inner three-bit counter, which counts clock provided by $10 \mathrm{MHz}$ oscillator in the rising edge of it. Data: input port of data, namely input port of base-band signal. Fsk_out: output port of FSK modulation. When data is high, fsk_out is connected with four times divided frequency via select switch, and with eight times divided frequency when data is low. The modulation of FSK comes true.

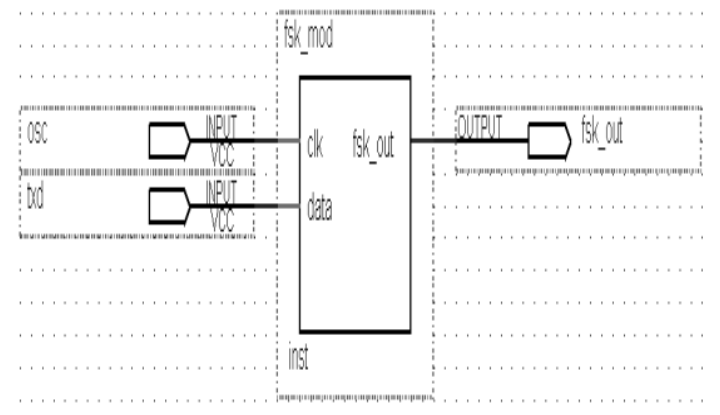

Fig.4 FSK modulation module

The logical structure of demodulation module is designed as shown in Fig.5. At the rise edge of the signal fsk_sig, the module de_fsk is triggered to save counter[7...0] to inner 8-bit register. Both they and synchronous signal are exported by tri_count. The module de_fsk2 is to assign tri_count to the two registers count 1 , count 2 in the role of synchronization signal and to export flag_out, which is used to judge the time sequences of two registers. In the module de_fsk3, the signal flag_out make count 1 minus count 2 , and the effect of counter reset is eliminated by algorithm to obtain the period of FSK. The output of de_fsk is ' 1 ' or ' 0 ' according to the period.

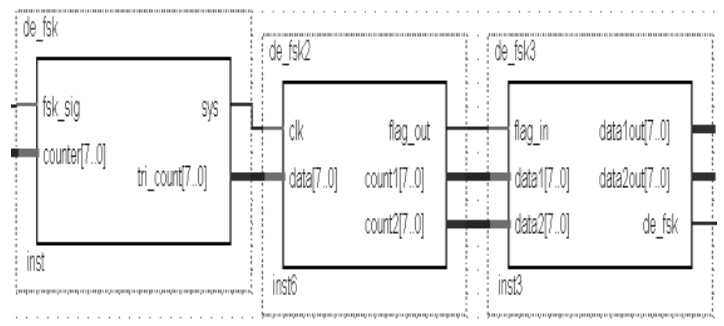

Fig.5 FSK demodulation module

\section{IV . Experimental Test}

The simulation based on Quartus II software is shown in Fig.6, where osd represents the input of clock with frequency $10 \mathrm{MHz}$, txd is data input port, fsk_out is FSK modulation output. From Fig.7, we can know that the frequency of fsk_out is $2.5 \mathrm{MHz}$ when data is ' 1 ' and $1.25 \mathrm{MHz}$ when data is ' 0 '. It means that the module of modulation can work in order.

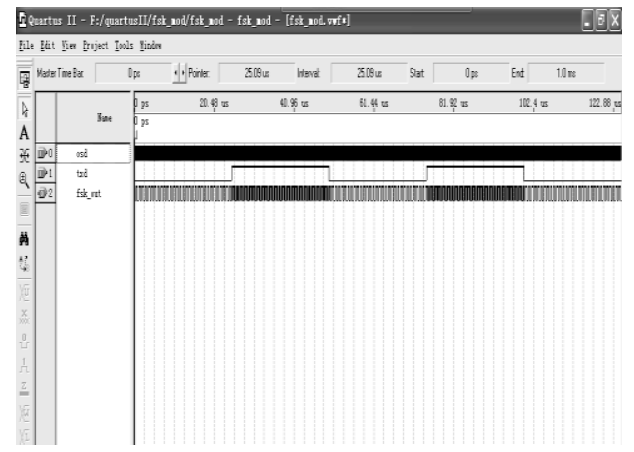

Fig.6 Simulation of FSK modulation module

Because the waveform simulation of Quartus II can't directly add FSK signal, all pins add in waveform simulation program in order to attach modulation module output to FSK input port of demodulation module. In this case, the simulations are shown in Fig.8, where osc represents the input of clock with frequency $10 \mathrm{MHz}$, txd is input port of FSK modulation module, de_fsk is FSK demodulation output. The modulation signal of modulation module is interiorly connected with input port of FSK demodulation module.

From Fig.7, we can know that the signal demodulated is the same with the corresponding input, and it means that the module of demodulation can work in order. However, race hazard will happen in the procedure of demodulation, so there are many flashes in the demodulated signal. It is necessary to design a filter module after demodulation to clear these flashes. The fabricated circuit of FSK modulation and demodulation is shown in Fig.8, which is composed of two pieces of circuit broads with CPLD. One is used for FSK modulation and other is for FSK demodulation. They are connected together by a triaxial cable. 


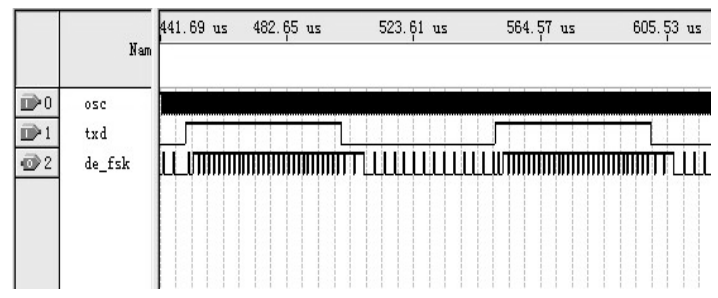

Fig.7 Simulation of FSK demodulation module

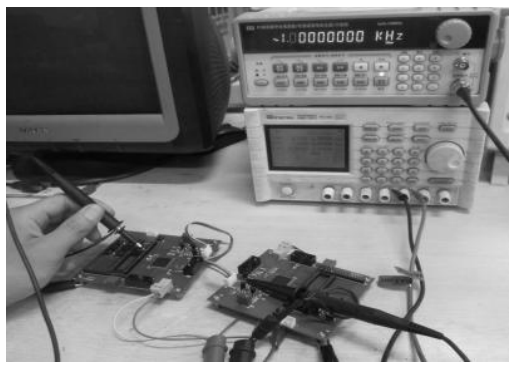

Fig.8 Fabricated circuit of FSK modulation and demodulation based on CPLD

A multi-function signal generator creates a square wave with the frequency of $10 \mathrm{kHz}$, the peak-to-peak amplitude of $3.3 \mathrm{~V}$, the DC bias of $1.65 \mathrm{~V}$, which is introduced into the baseband input port of CPLD. The test results about FSK modulation and demodulation are observed by oscilloscope as shown in Fig.9, where the waveform of the first channel is FSK modulation signal and that of the second channel is the signal via demodulation and filter based on CPLD. The RCtype filter with cut-off frequency of $40 \mathrm{kHz}$ can smooth the demodulation signal. The data via demodulation and filter is the same with the modulation signal. Experimental tests prove that the highest frequency of modulation signal based on CPLD can arrive at $15 \mathrm{kHz}$. It means that its baud rate with 30kbit/s can come true, whose speed is determined by exchange rate between SPI port of single-chip and USART.

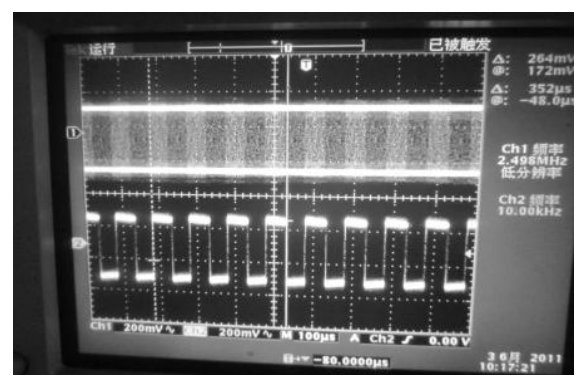

Fig.9 Experimental results of FSK modulation and demodulation

\section{Conclusion}

Two kinds of schemes of FSK modulation and demodulation based on single-chip and CPLD are compared and analyzed. The whole project FSK modulation and demodulation based on CPLD based on is put forward. A fulldigital circuit of FSK modulation and demodulation based on MAXII serial CPLD is designed, and the program of each module is written and validated by simulation. The experimental tests on the circuit show that it can work in order with these advantages of simple structure, good reliability and strong capability of anti-jamming.

\section{VI . Acknowledgment}

This work was supported by the National Nature Science Foundation of China under Grant Nos 61004130, the Fundamental Research Funds for the Central Universities under Grant Nos HUECFL20111107, Postdoctoral Science Foundation of China under Grant Nos 2012M511446 and 2013M530145

\section{References}

[1] FANG Yan-jun, SUN Jun, ZHU Xiao-ping. FSK modulator-demodulator based on.Instrument Technique and Sensor, 2008,7: 60-61,64.

[2] Bassel F, Chalres L. Asynchronous Classification of MFSK Signals Using the Higher Order Correlation Domain. IEEE Transactions on Communications, 1998, 46(4): 480 - 493.

[3] YING Ya-ping, XU Jian-feng, CHEN Wang-jun. Design and implementation of 2FSKmodulation-demodulation system based on FPGA. Journal of Zhejiang University of Technology, 2010,38(3): 282285.

[4] Antti Veikko Rosti, Visa Koivunen. Classification of MFSK modulated signals using the mean of complex envelope//Proc. EUSIPCO-2000, Tampere, Finland, 2000: 581-584.

[5] Bin Le, Thomas W.Rondeau, David Maldonado, et al. Modulation identification using neural networks for cognitive radios// IEEE 18th International Symposium on Personal, Indoor and Mobile Radio Communications, Athens , 2007: 1-5

[6] B. G. Mobasseri. Constellation shape as a robust signature for digital modulation recognition. IEEE Conf. of Military Communications, 1999: 442-446.

[7] S.-Z. Hsue and S. S. Soliman. Automatic modulation classification using zero crossing. IEEE Proc. Radar and Signal Processing, 1990, 137: 459464

[8] G. Arulampalam, V. Ramakonar, A. Bouzerdoum, and D.Habibi. Classification of digital modulation schemes using neural networks. IEEE Proc. Signal Processing and Its Applications, 1999,2: 45-53 